\title{
Validação de conteúdo do teste de conhecimento tático declarativo para o futsal
}

\section{Content validation of the declarative tactical knowledge test for futsal \\ Validez de contenido del test de conocimiento táctico declarativo para el fútbol de salón}

Fabiola de Araujo Cabral ${ }^{*}$ (D), Juan Carlos Pérez Morales ${ }^{b}$ (D),

Karen Cristine Rodrigues Alves ${ }^{a}$ (D) , Gibson Moreira Praça ${ }^{a}$ (i), Pablo Juan Greco ${ }^{a}$ (D)

Palavras-chave:

Validade de

conteúdo;

Conhecimento tático;

Avaliação;

Futsal.

\section{Keywords:}

Content validation;

Tactical knowledge;

Assessment;

Futsal.

\begin{abstract}
RESUMO
Objetiva-se demonstrar os indícios de validade de conteúdo de 125 cenas de ataque que foram extraídas de partidas oficiais de futsal masculino para compor o Teste de Conhecimento Tático Declarativo para o futsal (TCTD-FS). Cinco treinadores de futsal com no mínimo 10 anos de experiência avaliaram as cenas no que se refere à qualidade e pertinência, bem como a melhor tomada de decisão e sinais relevantes. Recorreu-se ao Coeficiente de Validade de Conteúdo (CVC), Concordância Entre Observadores (CEO), análise de conteúdo e validade ecológica. Os resultados indicaram 21 cenas com valores médios de $C V C>0,90$ e $C E O=1,0$. Conclui-se que as cenas selecionadas apresentaram evidências de validade de conteúdo e poderão compor a versão preliminar do TCTD-FS.
\end{abstract}

\begin{abstract}
The aim is to demonstrate the evidence of content validation for 125 offensive scenes extracted from official men's futsal matches to compose the declarative tactical knowledge test for futsal (TCTD-FS). Five coaches with at least 10 years of experience evaluated the scenes in terms of quality and relevance, the best tactical decision, and relevant cues. The Content Validity Coefficient (CVC), the agreement between observers (AO), the content analysis, and the ecological validity were used. The results indicated 21 scenes with mean values of CVC $>0.90$ and $A O=1.0$. It is concluded that the selected scenes present content validity and may compose the preliminary version of the TCTD-FS.
\end{abstract}

\section{RESUMEN}

Demostar los indícios de validez de contenido de 125 escenas de ataque extraídas de partidos oficiales de fútbol de salón masculino para hacer parte del test de conocimiento táctico declarativo para el fútbol de salón (TCTD-FS). Cinco entrenadores con al menos 10 años de experiencia evaluaron las escenas en términos de calidad y relevancia, selección de la mejor toma de decisión y pistas relevantes. Se utilizó el coeficiente de validez de contenido (CVC), el acuerdo entre observadores (AEO), el análisis de contenido y la validez ecológica. Los resultados determinaron 21 escenas con valores medios de CVC $>0.90$ y AEO $=1.0$. Se concluye que las escenas seleccionadas presentan evidencias de validez de contenido y se incluirán en la primera versión del TCTD-FS. 


\section{INTRODUÇÃO}

Nos esportes coletivos, como o futsal, o jogador na função de decisor procede à realização da sua ação com recurso à aplicação de uma técnica. Conforme as diversas tarefas que o jogador deve realizar no decorrer de uma partida, o processo de tomada de decisão se concretiza a partir da relação estabelecida com o ambiente, ou seja, a situação de jogo, e o nível de competência percebido. Discute-se esse processo de decisão à luz da teoria da ação (Nitsch, 2009), e dos sistemas dinâmicos, especificamente da denominada "abordagens dos constrangimentos" (Araújo et al., 2006, 2019). Tais teorias determinam que a natureza do comportamento humano radica na intenção da conduta dentro do contexto situacional, em que se exploram constrangimentos físicos e informacionais. Portanto, o jogador decide o que fazer e como fazê-lo adaptando seu comportamento conforme o ambiente de desempenho. No decorrer dessa tomada de decisão, os sistemas de controle cognitivo, emocional e automático, possibilitam que o jogador planeje, controle e avalie suas ações (Nitsch, 2009; Williams et al., 2011).

Nesse contexto, o conhecimento tático se apresenta como elemento fulcral no processo decisório referente aos esportes coletivos como o futsal. O conhecimento é definido como a informação representada e armazenada na memória que subjaz aos processos cognitivos (percepção, atenção, antecipação, tomada de decisão, dentre outros), classificado em conhecimento declarativo e processual (Sternberg, 2008). O conhecimento declarativo abrange fatos que podem ser declarados, "saber o quê" e o conhecimento processual se expressa em ações de habilidade "saber como fazer" (Ryle, 2009; Sternberg, 2008). Portanto, os desafios do processo de formação de esportistas, independente dos níveis de rendimento, estão ancorados na aquisição de Conhecimento Tático Declarativo (CTD) e Conhecimento Tático Processual (CTP) (Williams et al., 2011), e na adequada forma de mensurar e avaliar esse construto.

Diferentes modalidades como badminton (Blomqvist et al., 2000), futebol (Silva et al., 2018), handebol (Greco, 1995), tênis (Aburachid e Greco, 2010) e voleibol (Costa et al., 2016; Matias e Greco, 2009) possuem instrumentos validados em seu conteúdo para mensurar o CTD dos seus praticantes. Nesses procedimentos em geral, o CTD é analisado por meio da utilização de questionários ou de cenas de vídeo extraídas de partidas oficiais, em que se solicita ao avaliado elencar, seja de forma escrita ou verbal, as possíveis soluções para as diversas situações de jogo apresentadas. A justificativa para cada uma das soluções elencadas pelo avaliado se apoia na identificação dos sinais relevantes que devem ser percebidos e que orientam o processo de tomada de decisão. No caso específico do futsal, no melhor do nosso conhecimento, a literatura reporta dois estudos (Balzano e De Oliveira, 2013; Macedo, 2015) e duas produções referentes aos estudos de mestrado de Souza (2002) e Ribeiro (2013), em que se descreveu o procedimento de construção dos instrumentos utilizados para mensuração do CTD no futsal. Balzano e De Oliveira (2013) avaliaram o CTD em protocolos de vídeo nos quais os participantes deveriam identificar o sistema tático apresentado e selecionar a ação tática-técnica mais adequada que o jogador no ataque e na defesa deveria realizar para resolução da situação do jogo proposta. Macedo (2015), amparado no mesmo paradigma, avaliou o CTD a partir de um questionário com quatro imagens que representam situações táticas básicas no futsal, em que se solicita ao avaliado elencar a melhor ação táticatécnica que o jogador deve realizar conforme a situação do jogo apresentada.

Considerando que o conhecimento tático declarativo é um construto psicológico, espera-se que os testes sejam construídos a partir de paradigmas da psicometria. Contudo, verifica-se que os estudos de Balzano e De Oliveira (2013) e Macedo (2015) não descreveram detalhadamente como se procedeu à obtenção de evidências de validade de conteúdo, construto ou confiabilidade dos instrumentos propostos, conforme recomenda a literatura especializada na área da psicometria (Cicchetti, 1994; Pasquali, 2010). Relata-se apenas que as perguntas-itens e imagens que compõem os instrumentos passaram pela análise de especialistas na área, sem a utilização de procedimentos objetivos como o Coeficiente de Validade de Conteúdo (CVC) (Hernández-Nieto, 2002), o "V" de Aiken (Aiken, 1985) ou Concordância Entre Observadores (CEO). Além disso, não há indícios de confiabilidade dos instrumentos. Desta forma, não é possível determinar se as medidas obtidas por meio desses testes se referem ao construto psicológico que pretendem mensurar, o que se configura como uma desvantagem nesses instrumentos.

Nas propostas de mensuração do CTD de Souza (2002) e Ribeiro (2013) respectivamente, foram selecionadas cenas de ataque posicional 3-1 e de contra-ataque, extraídas de partidas oficiais de futsal. Ambos estudos analisaram a validade de conteúdo no processo de desenvolvimento dos instrumentos. Contudo, verifica-se que o instrumento proposto por Souza (2002) estaria desatualizado devido à alteração da regra do futsal no ano de 2011, que permitiu a utilização do goleiro como o quinto jogador de linha no campo ofensivo durante $o$ ataque. A utilização do goleiro no ataque para obtenção de superioridade numérica (5x4) é uma manobra frequentemente utilizada pelos técnicos para enfrentar os momentos críticos do jogo (Méndez-Domínguez et al., 2017). Por outro lado, embora o sistema ofensivo 3-1 seja frequentemente utilizado durante as partidas de futsal, é evidente que na interação ataque-defesa, as equipes de futsal utilizem diversos sistemas táticos ofensivos que possibilitem a conservação da posse de bola, a progressão para o objetivo, criação de desequilíbrio defensivo e oportunidades de finalização (Silva et al., 2011). Ao mesmo tempo, o instrumento proposto por Ribeiro (2013) apresentou também uma limitação no que se refere à representatividade do 
construto que se pretende mensurar (CTD), visto que no processo de construção, foram selecionadas cenas de jogos que contêm apenas situações de contra-ataque. Constata-se que $61,8 \%$ dos gols em uma partida de futsal se originam tanto no contra-ataque quanto no ataque posicional, bem como por meio da utilização do goleirolinha (Voser et al., 2016).

Conforme exposto, entende-se que seja oportuno iniciar o processo de construção e de obtenção de evidências de validade de um novo instrumento para mensuração do construto psicológico referente ao CTD no futsal. Objetiva-se com a proposição desse novo instrumento contribuir para a identificação de diferenças entre atletas de distintos níveis competitivos, categorias e tempo de prática no futsal. Afonso et al. (2012) afirmaram que a perícia dos atletas de jogos esportivos coletivos com maior nível de expertise está relacionada à sua competência para tomar decisões, a qual se apoia no conhecimento tático (declarativo e processual). Assim, "atletas experts" tomam decisões mais rápidas e com maior probabilidade de sucesso, ou seja, realizam escolhas corretas conforme o contexto situacional do jogo (Raab e Laborde, 2011).

Neste estudo, objetivou-se demonstrar os indícios de validade de conteúdo do teste de CTD para o futsal. Para tal, verificou-se se as cenas de ataque extraídas de partidas oficiais de futsal para compor o instrumento representaram comportamentos que definem o construto psicológico que se pretende mensurar (CTD).

\section{MATERIAIS E MÉTODOS}

\section{PROCEDIMENTOS ÉTICOS}

Este estudo respeitou as normas estabelecidas pelo Conselho Nacional de Saúde (CNS), sendo aprovado pelo Comitê de Ética sob o parecer № 44404515.1.0000.5149. Todos os participantes assinaram um Termo de Consentimento Livre e Esclarecido (TCLE).

\section{AMOSTRA}

Participaram cinco treinadores, peritos, na modalidade futsal com média de idade de $41,4( \pm 5,4)$ anos. Determinou-se como critério de inclusão, para assim ser considerado perito, ter no mínimo 10 anos de prática como treinador (Ericsson et al., 1993) e participação em campeonatos de nível nacional e/ou internacional (Tabela 1). Todos os treinadores possuíam títulos nesses níveis.

\section{PROCEDIMENTOS}

Para construção e validação de conteúdo das cenas inicialmente selecionadas para compor o Teste de Conhecimento Tático Declarativo para o Futsal: FS (TCTD-FS), seguiram-se os procedimentos ou passos propostos por Pasquali (2010).

\section{PRIMEIRO PASSO: SISTEMA PSICOLÓGICO}

Refere-se à definição do construto psicológico que o TCTD-FS pretende mensurar. O CTD se refere ao saber "o que fazer", ou seja, a escolha de alternativas táticas adequadas para solução dos diversos problemas do jogo, além do conhecimento sobre as regras da modalidade esportiva em questão, as posições dos jogadores e as estratégias básicas de defesa e ataque (Thomas et al., 1986).

\section{SEGUNDO PASSO: PROPRIEDADES}

O construto CTD foi representado mentalmente em termos de chunks (Anderson, 2014) que são unidades compostas de vários elementos no armazenamento da memória. Trata-se de um processo dinâmico, ativo e complexo, por meio da experiência, que permite ao cérebro lidar com memórias e ações complexas organizando-as em pequenos módulos ou sequências. No caso específico do futsal, os chunks permitem que o jogador estruture o conhecimento por meio de unidades de informação com diversos padrões. Esse processo possibilita tomar decisões mais rápidas e precisas na resolução dos diversos problemas do jogo.

\section{TERCEIRO PASSO: DIMENSIONALIDADE}

No processo de construção do TCTD-FS foram selecionadas cenas da fase de jogo de ataque devido que o principal objetivo do jogo de futsal é marcar o gol (Silva et al., 2011). Portanto, o TCTD-FS apresenta uma característica unidimensional denominada de ataque. A fase de jogo de ataque é classificada em ataque posicional e contra-ataque. No futsal, o ataque posicional se refere as ações ofensivas frente a uma defesa organizada e agrupada atrás da linha da bola.

Tabela 1. Relação de peritos com o respectivo tempo de prática e nível competitivo.

\begin{tabular}{ccc}
\hline Perito & Tempo de Prática & Nível Competitivo \\
\hline 1 & 20 anos & Internacional \\
2 & 18 anos & Nacional \\
3 & 24 anos & Internacional \\
4 & 12 anos & Nacional \\
5 & 21 anos & - \\
\hline
\end{tabular}


Por sua vez, o contra-ataque se refere ao momento do jogo em que ocorre a transição defesa-ataque de forma rápida, com objetivo de superar a oposição adversária e marcar o gol (Gonçalves, 2015; Silva et al., 2011).

\section{QUARTO PASSO: DEFINIÇÕES}

Neste estudo, a definição constitutiva do construto CTD se referiu ao "o que fazer", fatos que podem ser declarados e descritos, o que favoreceu a possibilidade de escolha, constituída de um corpo organizado de informações factuais (Anderson e Libierie, 2012). A definição operacional do CTD se referiu à capacidade de o jogador verbalizar a respeito das próprias decisões e das justificativas que possibilitaram as diferentes escolhas (Furley e Memmert, 2010).

\section{QUINTO PASSO: OPERACIONALIZAÇÃO}

Nesta fase, um grupo de acadêmicos e estudantes de pós-graduação em educação física selecionaram 125 cenas de ataque posicional e de contra-ataque, que foram extraídas de 14 jogos da categoria adulto masculino referentes à copa do mundo de futsal 2012, do campeonato europeu de futsal 2012, da liga dos campeões de futsal 2012 e 2014 e supercopa da Espanha 2011 e 2012. A utilização de cenas de jogos da categoria adulto referiu-se à organização do jogo assim como ao padrão técnico-tático dos atletas, que mais se aproximaram da excelência na modalidade, o que possibilitou melhor visualização das ações de jogo pelos avaliados. Todas as cenas foram editadas de forma que fosse possível observar as ações prévias ao momento em que o jogador com a posse de bola deveria tomar uma decisão, por exemplo, finalizar, passar ou conduzir. A duração das cenas variou entre 6 e 10 segundos, e elas permaneceram congeladas por 4 segundos no momento da tomada de decisão do jogador com posse de bola. O critério adotado para o estabelecimento do período que a cena permaneceu congelada se apoiou no tempo estabelecido pela regra da modalidade esportiva para realização do tiro lateral, do tiro livre, do arremesso de meta, do tiro de canto e para o controle da bola por parte do goleiro. Ao mesmo tempo, estudos prévios que também mensuraram o CTD adotaram em média 4 segundos para o congelamento da imagem no momento em que o jogador no ataque com bola deveria tomar uma decisão (Castro et al., 2017; Memmert e Furley, 2007; Raab e Laborde, 2011). Após o período em que a cena ficou congelada, a tela ficou preta (oclusão da imagem). Por fim, a pesquisadora principal fez uma reunião com cada um dos peritos da modalidade para a realização do processo de avaliação das 125 cenas.

\section{SEXTO PASSO: ANÁLISE DOS ITENS}

Esta etapa constituiu-se da avaliação da qualidade e da pertinência do item (cena) em relação ao construto a ser avaliado. Para tal, recorreu-se ao cálculo do CVC (Hernández-Nieto, 2002), bem como foram realizadas a avaliação e seleção da melhor tomada de decisão e dos sinais relevantes de cada cena (de acordo com a opinião dos peritos), e estabeleceu-se a validade ecológica dos itens por meio da comparação da decisão dos peritos com a decisão tomada pelo atleta com posse de bola na situação de jogo selecionada.

\section{AVALIAÇÃO DA QUALIDADE E PERTINÊN- CIA DO ITEM}

Cinco peritos avaliaram as 125 cenas de vídeo editadas conforme os critérios de Clareza da Imagem (CI), de Pertinência Prática (PP) e de Representatividade do Item (RI) (Hernández-Nieto, 2002), similarmente ao conduzido em estudos realizados em diferentes modalidades esportivas no Brasil (Aburachid e Greco, 2010; Matias e Greco, 2009; Souza, 2002). Utilizou-se uma escala tipo Likert, de cinco (5) pontos com intervalos iguais dentre eles. Posteriormente, os peritos discriminaram as cenas em relação às situações que representam, ou seja, se foram cenas de contra-ataque ou de ataque posicional.

$\mathrm{O}$ critério $\mathrm{Cl}$ se refere a qualidade visual, a nitidez e a abrangência da cena. A PP indica que as cenas representam situações características e adequadas para a tomada de decisão em um jogo de futsal. Por outro lado, o critério RI determina se as cenas selecionadas, no que se refere ao conteúdo, possibilitam a mensuração do construto (Aburachid e Greco, 2011).

\section{AVALIAÇÃO E SELEÇÃO DA MELHOR TOMADA DE DECISÃO E SINAIS RELEVANTES}

As cenas que permaneceram no teste foram reenviadas a três dos cinco peritos convidados inicialmente para participar do estudo. Solicitou-se que declarassem as possíveis decisões para cada situação e as hierarquizassem determinando qual seria a melhor opção, a segunda melhor opção, a terceira melhor opção e assim sucessivamente. Solicitou-se também aos peritos que justificassem suas opiniões com base nos sinais relevantes observados em cada cena. As cenas nas quais os peritos concordaram em $100 \%$ em relação a melhor decisão permaneceram no teste. Posteriormente realizou-se a análise de conteúdo, pela técnica da análise temática, para estabelecimento do gabarito com as respostas fornecidas pelos peritos, relativo à melhor tomada de decisão e aos sinais relevantes que se destacam como importantes a serem observados nas cenas.

\section{VALIDADE ECOLÓGICA}

Comparou-se a melhor tomada de decisão declarada pelos peritos com a ação realizada pelo atleta no momento do jogo, conforme sugerido na literatura (Aburachid e Greco, 2011). Permaneceram no teste as cenas nas quais os peritos e atletas apresentaram a mesma decisão, ou seja, o perito declarou em concordância com a ação 
realizada pelo atleta. Apenas as cenas que tiveram $100 \%$ de concordância foram selecionadas.

\section{ANÁLISE ESTATÍSTICA}

Para verificar a qualidade e pertinência dos itens nos critérios $\mathrm{Cl}$, PP e RI, recorreu-se ao CVC desenvolvido por Hernández-Nieto (2002). Para tal, utilizaram-se as notas atribuídas pelos peritos conforme a equação apresentada no Quadro 1. Apenas as cenas com CVC $\geq 0,8$ foram selecionadas (Balbinotti, 2005).

1) Cálculo da média $(M x)$ : onde $\Sigma x_{l=}$ soma das notas dos peritos e $J$ = número de peritos;

2) Cálculo do CVC para cada item $\left(C V C_{i}\right)$ : onde $V_{\text {MÁx }}$ = valor máximo que o item poderia receber;

3) Cálculo do erro $\left(P_{\text {ei }}\right)$;

4) Cálculo do CVC final de cada item: $\left(\mathrm{CVC}_{\mathrm{c}}\right)$;

5) Cálculo do CVC total do instrumento para

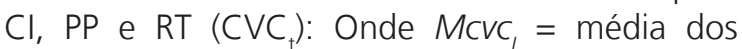
coeficientes de validade de conteúdo dos itens e Mpe $_{\iota}=$ média dos erros dos itens.

Posteriormente, considerou-se a CEO para se definir a situação de ataque a qual as cenas pertenciam. Além disso, considerou-se a objetividade das tomadas de decisões dos peritos para estabelecer, como critério, quais as melhores cenas que iriam compor a versão preliminar do teste (Aburachid e Greco, 2010). O cálculo do CEO corresponde à divisão entre o número de concordâncias entre observadores e a somatória das concordâncias e discordâncias. Adotou-se o valor de $\mathrm{CEO}=1,0$ de concordância para permanência das cenas.

Finalmente, procedeu-se à criação do gabarito do instrumento e estabelecimento da validade ecológica das cenas conforme descrito anteriormente nos passos para construção e validação do TCTD-FS.

\section{RESULTADOS}

A Figura 1 descreve o número total de cenas que iniciaram o processo de validação e a quantidade que permaneceu após o procedimento de validação de conteúdo.
Conforme se observa na Figura 1, a construção do instrumento foi iniciada com 125 cenas. Ao final do processo restaram 21 cenas, nove cenas de ataque posicional e 12 cenas de contra-ataque. Ressalta-se que as cenas descartadas alcançaram valores de CVC acima de 0,80 , porém tiveram que ser retiradas porque as mesmas não cumpriram com o critério de concordância entre observadores ou de validade ecológica.

A Tabela 2 descreve os valores de CVC obtidos a partir da avaliação dos peritos quanto a $\mathrm{Cl}$, PP e RI.

Para as cenas que permaneceram no instrumento após concluída a validade de conteúdo, obteve-se um valor de CVC médio acima de 0,90. Estes resultados representam que as cenas apresentaram adequados índices de validade de conteúdo para compor o teste.

\section{DISCUSSÃO}

Este estudo objetivou demonstrar os indícios de validade de conteúdo, de cenas de ataque posicional e de contra-ataque extraídas de partidas de futsal, no intuito de construir o TCTD-FS. Para tal, verificou-se se as cenas que puderam compor a versão preliminar do instrumento representaram comportamentos que definiram o construto que se pretendeu mensurar. Os cálculos do CVC (Hernández-Nieto, 2002) reportaram valores $>0,90$ para todas as 21 cenas selecionadas (nove cenas de ataque posicional e 12 cenas de contra-ataque)

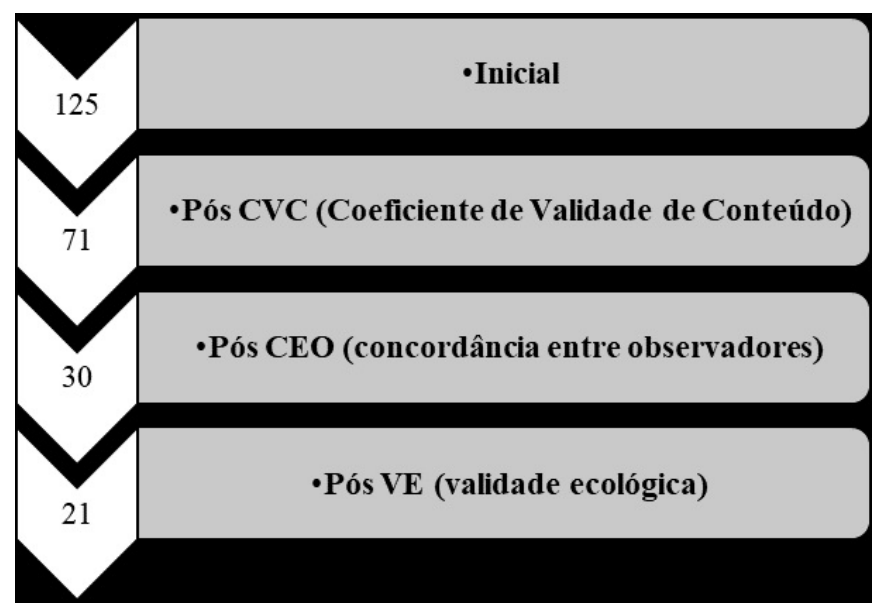

Figura 1. Relação da quantidade de cenas iniciais e finais após validação de conteúdo.

Quadro 1. Equação para o cálculo do CVC.

\begin{tabular}{|c|c|c|c|c|}
\hline $\mathbf{1}$ & $\mathbf{2}$ & $\mathbf{3}$ & $\mathbf{4}$ & $\mathbf{5}$ \\
\hline$\sum_{i=1}^{J} x_{i}$ & $C V C_{i}=\frac{M_{x}}{V_{\text {máx }}}$ & $P e_{i}=\left(\frac{1}{J}\right)^{J}$ & $C V C_{c}=C V C_{i}-P e_{i}$ & $C V C_{t}=M c v c_{i}-M p e_{i}$ \\
\hline
\end{tabular}

Fonte: Hernández-Nieto (2002). 
Tabela 2. Valores de CVC médio para as cenas que permaneceram e para as cenas que foram descartadas, em cada um dos critérios de avaliação.

\begin{tabular}{cccc}
\hline Cenas & Selecionadas & Descartadas & TOTAL \\
\hline Clareza de Imagem (CI) & 0,94 & 0,90 & 0,90 \\
Pertinência Prática (PP) & 0,97 & 0,95 & 0,95 \\
Representatividade do Item & 0,93 & 0,85 & 0,87 \\
(RI) & & & \\
\hline
\end{tabular}

nos quesitos $\mathrm{Cl}$, PP e RI. Assim, confirmam-se os indícios de validade do TCTD-FS.

O CVC foi utilizado em diferentes estudos acadêmicos como medida objetiva para determinar evidências de validade de conteúdo de instrumentos para mensuração do CTD no tênis (Aburachid e Greco, 2011), no voleibol (Costa et al., 2016) e no futebol (Silva et al., 2018). Os valores do CVC reportados para esses três instrumentos foram $\geq 0,80$, o que corrobora os resultados alcançados pelas cenas selecionadas para compor o instrumento proposto neste estudo. Na perspectiva do CTP, estudos na área de construção e validação de instrumentos para a orientação esportiva (Castro et al., 2015; Greco et al., 2015) e para o basquetebol (Pérez-Morales et al., 2018), recorreram ao CVC como procedimento para obtenção de evidências de validade de conteúdo dos instrumentos propostos. Nesses estudos, a $\mathrm{Cl}$ foi substituída pela clareza de linguagem, visto que ambos os instrumentos propunham a observação de comportamento táticotécnico em situações de jogo na configuração tática de $3 \times 3$. Os valores de CVC reportados para o instrumento referente à orientação esportiva, denominado de teste de conhecimento tático processual para orientação esportiva (TCTP-OE), foi $>0,80$. No que se refere ao instrumento proposto para o basquetebol, denominado teste de CTP para o basquetebol (TCTP-Bb), os valores de CVC foram $>0,90$. Ressalta-se que a literatura recomenda valores de $C V C \geq 0,80$, o que indica concordância e validade adequadas (Cassepp-Borges et al., 2010; Hernández-Nieto, 2002). Nesse sentido, entende-se que as cenas selecionadas para compor a primeira versão do teste de CTD para o futsal apresentaram evidências de validade de conteúdo suficientes, visto que os valores de CVC alcançados foram interpretados como excelentes (Hernández-Nieto, 2002).

No que se refere ao número total de cenas selecionadas após os procedimentos para obtenção de evidências de validade de conteúdo, a literatura na área da psicometria recomenda um mínimo de 20 itens validados para mensuração do construto (Pasquali, 2013). Considerando que o TCTD-FS apresenta uma característica unidimensional denominada de ataque, composta pelas situações de Ataque Posicional (AP) e de Contra-Ataque (CA), as 21 cenas selecionadas para compor o instrumento (nove de AP e 12 de CA) foram suficientes para representação da dimensão e mensuração do CTD de jogadores de futsal. Por esse motivo, não foi necessária a validação de no mínimo 20 cenas para cada uma das situações que compõem a dimensão ataque, isto é para o AP e o CA. Costa et al. (2016) validaram no seu conteúdo 66 cenas extraídas de jogos de voleibol referentes à dimensão ataque, sendo nove cenas de ataque na extremidade, sete de ataque central, 25 de levantamento e 25 de bloqueio. Nesse sentido, o número mínimo de cenas selecionadas no procedimento da validação de conteúdo do TCTD-FS pôde atender a dimensão que as cenas representaram e não as categorias que puderam compor a dimensão. Por fim, instrumentos propostos para mensuração do CTD no tênis (Aburachid e Greco, 2011), no basquetebol (Morales et al., 2017) e no futebol (Silva et al., 2018) validaram entre 11 e 20 cenas, o que sugere que o número de cenas selecionadas neste estudo está dentro das recomendações propostas tanto pela literatura quanto pelas evidências empíricas de estúdios prévios.

Este estudo apresentou indícios de validade de construto do TCTD-FS. Desta forma, recomenda-se a continuidade aos procedimentos de obtenção de evidências de validade de construto e confiabilidade, conforme foi proposto por Pasquali (2010). Ressalta-se que, além da mensuração do CTD sobre as 21 cenas validadas, no que se refere ao conteúdo, sugere-se sua utilização em estudos de intervenção que objetivem avaliar o impacto de programas de treinamento da atenção-percepção e de tomada de decisão no futsal. Estudos na área dos jogos esportivos coletivos reforçam a importância do treinamento da atenção e da percepção, por meio da utilização de cenas de jogos em que o jogador com a posse de bola deve tomar uma decisão, para aquisição do CTD (Alarcón et al., 2011; Pagé et al., 2019).

\section{CONSIDERAÇÕES FINAIS}

Conclui-se que o procedimento de obtenção de evidências de validade de conteúdo foi finalizado com uma quantidade suficiente de cenas para mensuração do construto CTD para a modalidade futsal. A utilização do CVC possibilitou a seleção de cenas de ataque extraídas de partidas oficiais de futsal com adequados níveis de concordância e validade. No que se refere às características das cenas selecionadas ao final dos procedimentos de validação de conteúdo, constatou-se adequada representatividade contextual da modalidade esportiva do futsal, visto que foi possível contar com 
cenas tanto de ataque posicional quanto de contraataque. Por fim, ressalta-se a importância de fornecer continuidade com os procedimentos de obtenção de evidências de validade de construto e confiabilidade.

\section{FINANCIAMENTO}

O presente trabalho contou com apoio financeiro da Pró-reitoria de Pesquisa (PRPq) da Universidade Federal de Minas Gerais (UFMG).

\section{CONFLITOS DE INTERESSE} interesse.

Os autores declaram não haver conflitos de

\section{REFERÊNCIAS}

Aburachid LMC, Greco PJ. Processos de validação de um teste de conhecimento tático declarativo no tênis. Rev da Educ Física. 2010;21(4):603-10. http://dx.doi.org/10.4025/ reveducfis.v21i4.8355.

Aburachid LMC, Greco PJ. Validação de conteúdo de cenas do teste de conhecimento tático no tênis. Estud Psicol. 2011;28(2):261-7.

Afonso J, Garganta J, Mesquita I. A tomada de decisão no desporto: o papel da atenção, da antecipação e da memória. Rev Bras Cineantropom Desempenho Hum. 2012;14(5):592-601. http://dx.doi.org/10.5007/1980$0037.2012 v 14 n 5 p 592$.

Aiken LR. Three coefficients for analyzing the reliability and validity of ratings. Educ Psychol Meas. 1985;45(1):131-42. http://dx.doi.org/10.1177/0013164485451012.

Alarcón FL, Cárdenas DV, Miranda MTL, Ureña NO, Piñar MIL. Influencia del programa de entrenamiento reflexivo sobre el conocimiento declarativo de un equipo de baloncesto. Cuad Psicol Deporte. 2011;11(1):19-28.

Anderson JR, Libierie C. The atomic components of thougth. New York: Psycology Press; 2012.

Anderson JR. Rules of the mind. New York: Psychology Press; 2014. http://dx.doi.org/10.4324/9781315806938.

Araújo D, Davids K, Hristovski R. The ecological dynamics of decision making in sport. Psychol Sport Exerc. 2006;7(6):653-76. http://dx.doi.org/10.1016/j. psychsport.2006.07.002.

Araújo D, Hristovski R, Seifert L, Carvalho J, Davids K. Ecological cognition: expert decision-making behaviour in sport. Int Rev Sport Exerc Psychol. 2019;12(1):1-25. http://dx.doi. org/10.1080/1750984X.2017.1349826.

Balbinotti MAA. Para se avaliar o que se espera: reflexões acerca da validade dos testes psicológicos. Aletheia. 2005;(21):43-52.

Balzano ON, De Oliveira EM. Proposta de avaliação do nível de conhecimento tático declarativo e tomadas de decisões, dentro das capacidades coletivas de ataque e defesa no futsal. Lect Educ Física y Deport. 2013;18(181):1.

Blomqvist $\mathrm{M}$, Luhtanen $\mathrm{P}$, Laakso L, Keskinen E. Validation of a video based game understanding test procedure in badminton. J Teach Phys Educ. 2000;19(3):325-37. http:// dx.doi.org/10.1123/jtpe.19.3.325.
Cassepp-Borges V, Balbinotti MAA, Teodoro MLM. Tradução e validação de conteúdo: uma proposta para adaptação de instrumentos. In: Pasquali L, editor. Instrumentação Psicológica: fundamentos e práticas. Porto Alegre: Artmed; 2010. p. 506-20.

Castro HO, Costa GCT, Praça GM, Campbell CSG, Greco PJ. Analysis of visual fixations and decision-making in volleyball athletes from infanto and juvenile categories. Rev Bras Ciênc Mov. 2017;25(1):51-9.

Castro HO, Morales JCP, Aburachid LMC, Greco PJ. Teste de conhecimento tático processual $3 \times 3$ com os pés: alternativa para a orientação esportiva. Rev Bras Educ Fís Esporte. 2015;29(4):621-9. http://dx.doi.org/10.1590/180755092015000400621.

Cicchetti DV. Guidelines, criteria, and rules of thumb for evaluating normed and standardized assessment instruments in psychology. Psychol Assess. 1994;6(4):28490. http://dx.doi.org/10.1037/1040-3590.6.4.284.

Costa GDCT, Castro HO, Cabral FDA, Morales JCP, Greco PJ. Content Validity of scenes of the Declarative Tactical Knowledge Test in Volleyball - DTKT:Vb. Rev Bras Cineantropom Desempenho Hum. 2016;18(6):629-37. http://dx.doi.org/10.5007/1980-0037.2016v18n6p629.

Ericsson K, Krampe R, Tesch-Rõmer $\mathrm{C}$. The role of deliberate practice in the acquisition of expert performance. Psychol Rev. 1993;100(3):363-406. http://dx.doi. org/10.1037/0033-295X.100.3.363.

Furley PA, Memmert D. The role of working memory in sport. Int Rev Sport Exerc Psychol. 2010;3(2):171-94. http:// dx.doi.org/10.1080/1750984X.2010.526238.

Gonçalves MC. Análise dos gols da segunda fase da liga futsal 2013. Rev Bras Futsal e Futeb. 2015;7(24):153-7.

Greco PJ, Perez Morales JC, Aburachid LMC, Silva SR. Evidência de validade do teste de conhecimento tático processual para orientação esportiva - TCTP: OE. Rev Bras Educ Fís Esporte. 2015;29(2):313-24. http://dx.doi. org/10.1590/1807-55092015000200313.

Greco PJ. O ensino do comportamento tático nos jogos esportivos coletivos: aplicação no handebol [tese]. Campinas: Universidade Estadual de Campinas; 1995.

Hernández-Nieto RA. Contributions to statistical analysis. Mérida: Universidad de Los Andes; 2002.

Macedo T. Comparação do conhecimento tático declarativo de jogadores de futsal das categorias de base. Rev Bras Futsal e Futeb. 2015;7(24):141-7.

Matias CJAS, Greco PJ. Desenvolvimento e validação do teste de conhecimento tático declarativo para o levantador de voleibol. Arq em Mov. 2009;5(1):61-80.

Memmert D, Furley P. "I spy with my little eye!": breadth of attention, inattentional blindness, and tactical decision making in team sports. J Sport Exerc Psychol. 2007;29(3):365-81. http://dx.doi.org/10.1123/ jsep.29.3.365. PMid:17876972.

Méndez-Domínguez C, Gómez-Ruano MA, Ruíz-Pérez LM, Cui $Y$. Unfavorable critical moments and way of facing them from the futsal coach's point of view through ad hoc questionnaire. Rev Int Cienc Deporte. 2017;13(50):331-55. http://dx.doi.org/10.5232/ricyde2017.05002. 
Morales JCP, Olliveira R, Praça GM, Greco PJ. Evidências de Validade de Conteúdo de Cenas de Ataque para Construção de um Teste de Conhecimento Tático Declarativo para o Basquetebol. In: Livro de Resumos do $6^{\circ}$ Congresso Internacional de Jogos Desportivos: Desafios da Excelência nos Jogos Desportivos Coletivos: da investigação à prática; 2017; Porto. Porto: Faculdade do Desporto da Universidade do Porto; 2017. p. 136.

Nitsch JR. Ecological approaches to Sport Activity: a commentary from an action-theoretical point of view. Int J Sport Psychol. 2009;40:152-76.

Pagé C, Bernier PM, Trempe M. Using video simulations and virtual reality to improve decision-making skills in basketball. J Sports Sci. 2019;37(21):2403-10. http://dx.doi. org/10.1080/02640414.2019.1638193. PMid:31280685.

Pasquali L. Psicometria: teoria dos testes na psicologia na educação. 5. ed. Petrópolis: Vozes; 2013.

Pasquali L. Testes referentes a construto: teoria e modelo de construção. In: Pasquali L, editor. Instrumentação Psicológica: fundamentos e práticas. Porto Alegre: Artmed; 2010. p. 165-98.

Pérez-Morales JC, Greco PJ, Ferreira-Lopes B, Estevão BJ, Ibañez SJ. Development and preliminary validation of a new Procedural Tactical Knowledge Test for Basketball using 3vs.3 situation. Rev Int ciencias del Deport. 2018;14(53):256-67.

Raab M, Laborde S. When to blink and when to think: preference for intuitive decisions results in faster and better tactical choices. Res Q Exerc Sport. 2011;82(1):8998. http://dx.doi.org/10.1080/02701367.2011.1059972 5. PMid:21462689.
Ribeiro DA. Tomada de Decisão e Conhecimento Declarativo de futsalonistas adultos de diferentes níveis competitivos em situação de contra-ataque [dissertação]. Londrina: Universidade Estadual de Londrina; 2013.

Ryle G. The concept of Mind. New York: Routledge Taylor \& Francis Group; 2009. http://dx.doi.org/10.4324/9780203875858.

Silva JVO, Greco PJ, Morales JCP, Castro HO, Costa GCT, Praça GM. Declarative and procedural tactical knowledge in soccer: analysis in U-14 and U-15 youth players. J Phys Educ (Maringá). 2018;29(1):1-10. http://dx.doi.org/10.4025/ jphyseduc.v29i1.2974.

Silva MV, Ré AHN, Matias CJAS, Greco PJ. Estratégia e Tática no Futsal: uma análise crítica. Cad Educ Física. 2011;10(19):75-84.

Souza PRC. Validação de testes para avaliar a capacidade de tomada de decisão e o conhecimento tático declarativo em situações de ataque no Futsal [dissertação]. Belo Horizonte: Universidade Federal de Minas Gerais; 2002.

Sternberg RJ. Psicologia cognitiva. 4. ed. Porto Alegre: Artmed; 2008.

Thomas JR, French KE, Humphries CA. Knowledge development and sport performance: directions for motor behavior research. J Sport Psychol. 1986;8(4):259-72. http://dx.doi. org/10.1123/jsp.8.4.259.

Voser RC, Silva CG, Voser PEG. A origem dos gols da Liga de Futsal 2014. Rev Bras Futsal e Futeb. 2016;8(29):155-60.

Williams AM, Ford PR, Eccles DW, Ward P. Perceptual-cognitive expertise in sport and its acquisition: implications for applied cognitive psychology. Appl Cogn Psychol. 2011;25(3):432-42. http://dx.doi.org/10.1002/acp.1710. 